\title{
Designing primers potentially specific to Entamoeba gingivalis genes
}

\begin{abstract}
JAgODA ABrameK
Chair and Department of Biology and Genetics, Faculty of Pharmacy; Medical University of Lublin; Chodzki 4a, 20-093 Lublin, Poland
\end{abstract}

\begin{tabular}{|c|c|}
\hline ARTICLE INFO & ABSTRACT \\
\hline $\begin{array}{l}\text { Received } 01 \text { July } 2015 \\
\text { Accepted } 20 \text { August } 2015\end{array}$ & \multirow{2}{*}{$\begin{array}{l}\text { Entamoeba gingivalis normally exists in the human oral cavity, namely in the gums, and } \\
\text { brings about some specific diseases. However, it can also trigger some more serious } \\
\text { illnesses. Among these are infections of the genital tract, acute osteomyelitis of the } \\
\text { mandible and pulmonary abscess. Entamoeba gingivalis identification by light microscopy } \\
\text { is difficult, hence polymerase chain reaction (PCR) is used. The contemporary primers } \\
\text { for PCR are complement to } 18 \mathrm{~S} \text { rRA. This article informs the reader of the process } \\
\text { that was involved in designing new primers for three genes which were thought to be } \\
\text { present on the Entamoeba gingivalis genome, but their sequences were unknown. The } \\
\text { newly obtained sequences of primers have better properties for identification purposes, } \\
\text { compared to these which are currently used. }\end{array}$} \\
\hline $\begin{array}{l}\text { Keywords: } \\
\text { actin, } \\
\text { cysteine proteinase, } \\
\text { Entamoeba gingivalis, } \\
\text { 18S-rRNA, } \\
\text { 5.8S-rRNA, } \\
\text { PCR primers. }\end{array}$ & \\
\hline
\end{tabular}

\section{INTRODUCTION}

Entamoeba gingivalis is a protozoan which can be found in the gums and around the teeth. It exists solely as a trophozoite and does not produce any cysts [3]. Currently, researchers are unsure whether it is a parasite, but its occurrence is connected with diseased gingival pockets [10]. Humans can be infected as a result of direct oral contact or by sharing dishes or cutlery. Entamoeba gingivalis has been also found in cases of more serious nature such as acute osteomyelitis of the mandible [1] and in pulmonary abscess [6] in the elderly. It is also found in the genital tract [5]. Entamoeba gingivalis can be identified by light microscopy, but it bears high resemblance to other Entamoeba species. Polimerase Chain Reaction (PCR) is a more specific and sensitive method. The Entamoeba gingivalis genome has not been fully sequenced yet, and the 18S rRNA gene is the only gene of this protozoa with known sequence, and therefore, it is used in PCR [10]. Primers to other genes of Entamoeba gingivalis can be designed on the basis of sequence resemblance to closely related species, such as pathogenic Entamoeba histolytica, non-pathogenic amoebae Entamoeba dispar and Entamoeba moshkovskii, as well as more distant species of amoeba, such as Acanthamoeba castellanii. Candidate genes for this case are cysteine proteinase, actin and 5.8SrRNA,

\footnotetext{
* Corresponding author

e-mail: jagoda.abramek@umlub.pl;

tel. 814487236
}

because sequences of the genes from different amoebae species are available in the GenBank Database.

The cysteine proteinases present in amoebae are connected with invasion and host tissue penetration because they are involved in cleaving the extracellular matrix. They also interfere with the immune system of the host, e.g. by complement and antibodies IgA and IgG degradation. Entamoeba histolytica produces a large amount of different cysteine proteinases which correlate with the invasion. Entamoeba dispar which is a very similar, though nonpathogenic amoeba, also produces some cysteine proteinases, but in smaller amounts [8]. Actin, which is a protein present in most eukaryotic cells, is highly conserved among the species, and takes part in maintaining cell shape [4], whereas, $5.8 \mathrm{~S}$ rRNA is a type of non-coding rRNA which is a component of the large subunit of the eukaryotic ribosome, and so plays a role in protein translation [2].

\section{MATERIALS AND METHODS}

Primer design. In order to design sets of primers for genes such as cysteine proteinase, actin and 5.8S rRNA, the GenBank sequences of these genes from closely related protozoa were used. In this work, sequences of each gene were aligned along one another using Clustal X. Subsequently, the potential primers were designed using Primer 3 program to be complement to the most conservative regions of each gene [9]. The primers specificity was then tested 
using the Primer-BLAST program. Next, the OligoAnalyser program was used to determine primer properties such as the possibility of hairpin, self-dimer and hetero-dimer formation. On this basis, one pair of the best primers for each gene was chosen.

\section{RESULTS}

Primer design. GenBank cysteine proteinase gene sequences from Entamoeba histolytica (gi|1246522) and
Entamoeba invadens (gi|881587), as well as mRNA from Entamoeba dispar (gi|1246518) were aligned.

ed_cpr
eh_cp
ei_cp
ed_cpr
eh_cp
ei_cp
ed_cpr
eh_cp
ei_cp
ed_cpr
eh_cp
ei_cp
ed_cpr
eh_cp
ei_cp
ed_cpr
eh_cp
ei_cp
eh_cp
ei_cp
ed_cpr
eh_cp
ei_cp
ed_cpr_cp
ed_cpr
eh_cp
ei_cp

AAAGATTGGAGAgCTGAAGGTAAAGTTACTCCAGTTAGAGATCAAGGAAATTGTGGATCA AAAGATTGGAGAGCTGAAGGTAAAGTTACTCCAGTTAGAGACCAAGGAAATTGTGGATCA GTTGATTGGAGAAAAGAAGGAAAAGTAACTCCAATTAGAGATCAAGCACAATGCGGATCA

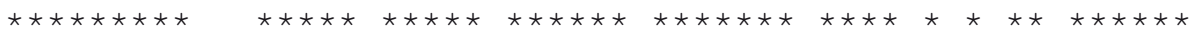
TGTTATTCATTCTCATCACTTGCTGTCCTTGAATCAAGATTATTAATTGCTGGAAGCAAA TGCTATTCATTCTCATCACTTGCTGTTCTTGAATCAAGATTATTAATTGCTGGAAGCAAA TGTTATACATTTGGTTCACTTGCAGCTCTTGAAGGAAGATTATTAATTGA-AAAAGGAGG

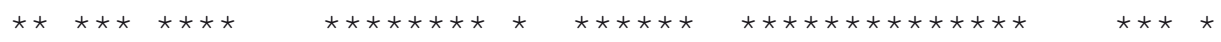

TACAACCAAAACAATCAAGATCTTTCAGAACAACAAATTGTTGACTGTA------GTACT TACAACCAAAACAACCTTGATCTTTCTGAACAACAAATTGTTGATTGTA------GTGCT TGATGCTAATACA--CTCGATCTT-CAGAAGAACATATGGTTCAATGCACAAGAGATAAT

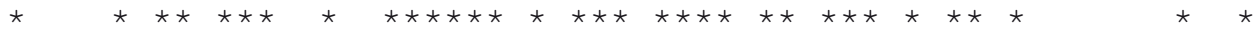
GCTAATAATGGATGTAATGGAGGATCTCTCTCTGCTACTTATCTTTATGTTAA-AAATAA GCTAATAATGGATGTAATGGAGGATCTCTTTCTGCTACTTATCTTTATGTTAA-AAATAA GGAAATAATGGATGTAATGGAGGACTTGGATCAAATGTCTATGATTACATTATTGAACAC

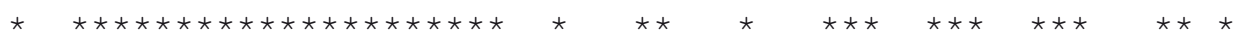
TGGTGTTACTGATGAAGCTTCATACCCATACACAGCTACTAAGGGAACTTGCAAAGCTTAGGTGTTACTGATGAAGCCTCATATCCATACACTGCTACTAAGGGAACTTGTAAAGCTTGAGTGTCA---AAGAAAGTGATTATCCATACACTGGAAATGATTCTACATGCAAAACTAA

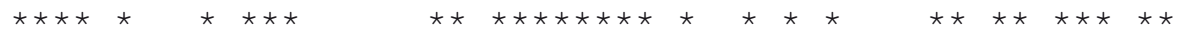

--TCACTCCAAAAGTTCAAACTACTGGTTTAACTCATGTCACTCCAAATGAAGATGCT---TCACTCCAAAAGTTCAAACTACTGGTTTAACTCATGTTACTCCAACTGAAGAAGCT-TGTAAAATCATTTGCTAAAATTACTGGATATACTAAAGTCCCAAGAAACAATGAAGCTGA

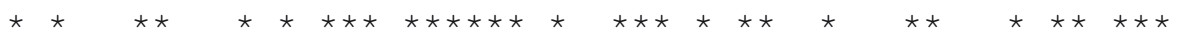

-TTGACTTCAGCTCTTGAACAAGGACCAGTTGCTGTTTGTATTGATGCTGGTAAAGCTTC -TTAACTGCTGCTCTTGCAGAAGGACCAGTTGCTGTTTGTATTGATGCTGGTAAAGCTTC ACTTAAAGCTGCACTTTCACAAGGTCTTATTGATGAAACAAATGATGCATCATCTGCTAA

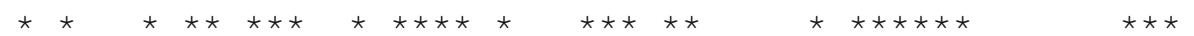
ATTCCAATTATATAAATCTGGAGTTTATGATGAACCAAAATGTAGTAA-AACTGTT---ATTCCAATTATATAAAAATGGAGTTTATGATGAACCAAAATGCAGCAA-AACTGTT---ATTCCAATTATACAAGAGCGGAGCTTATACTGATACTAAATGCAAGAATAACTACTTTGC

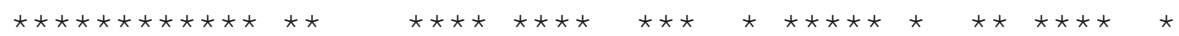
--- AACCATGGTGTCGCCGCTGTTGGATATGGTACTCAAGATGGTAAAGACTATTATAT ----AACCATGGTGTTGCCGCTGTAGGATATGGATCTCAAGATGGTCAAGACTATTACAT TTTGAATCACGAAGTTTGTGCTGTTGGATATTGTGTTGTTGATGGAAAAGAATGTTGGAT

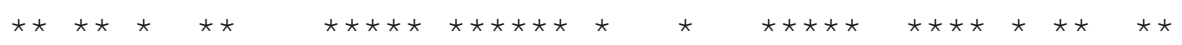
TGTTAAGAACTCATGGGGAACTTCATGGGGAGACAAAGGTTATATTTTAATGTCAAGAAA TGTTAAGAACTCATGGGGAACTTCATGGGGAGATAAAGGTTATATTTTAATGTCAAGAAA

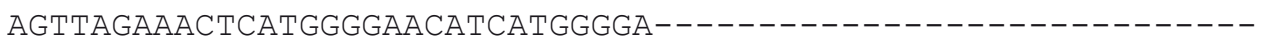

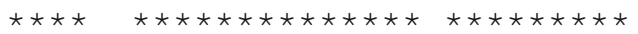

Figure 1. CLUSTAL 2.1 multiple sequence alignment of the cysteine proteinase gene 


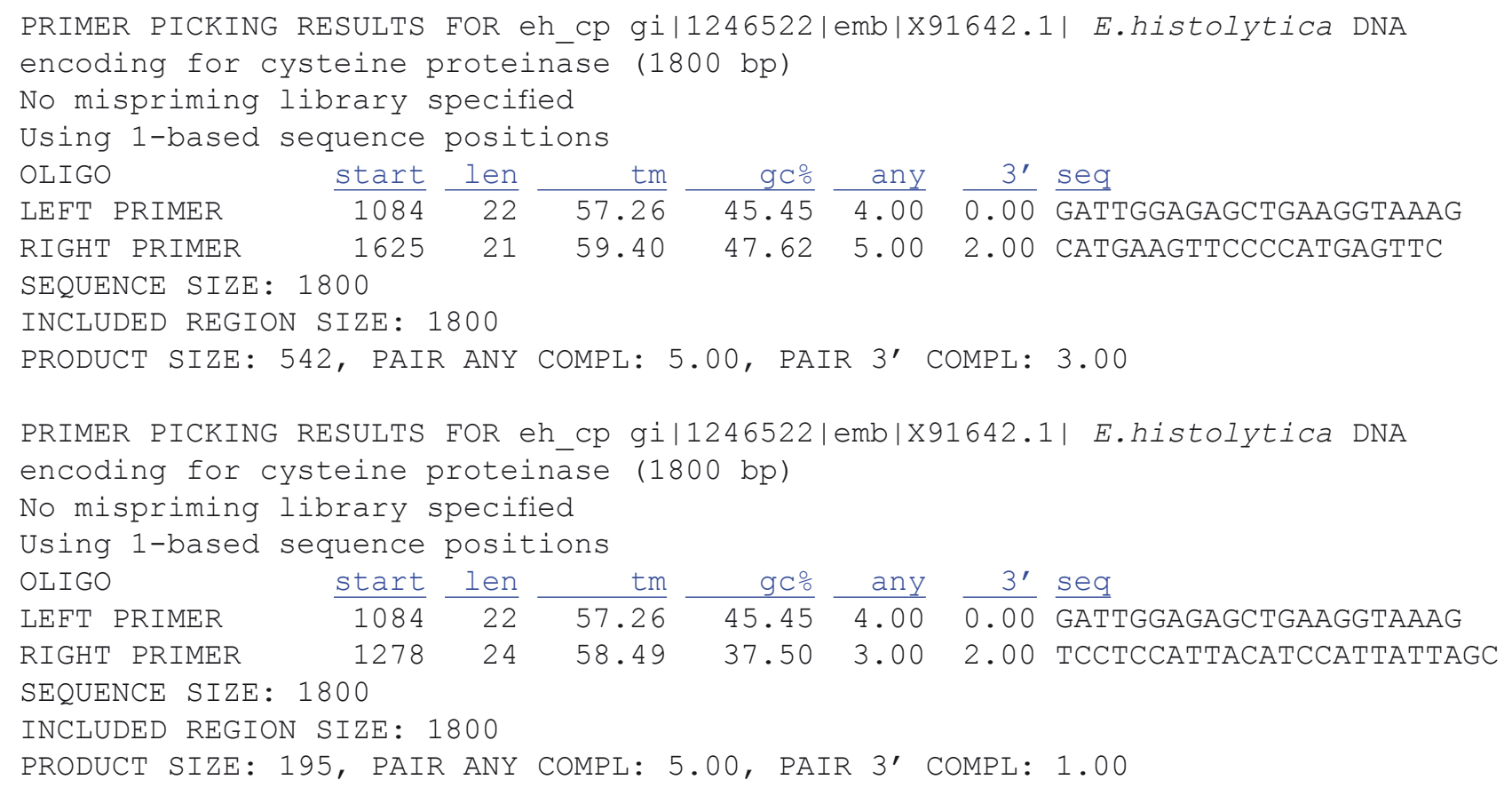

Figure 2. Two primer pairs generated using the Primer 3 program

The specificity of the generated primers was confirmed using Primer-BLAST. The GC content of the second pair of primers is below $40 \%$, hence, it is not taken into account.

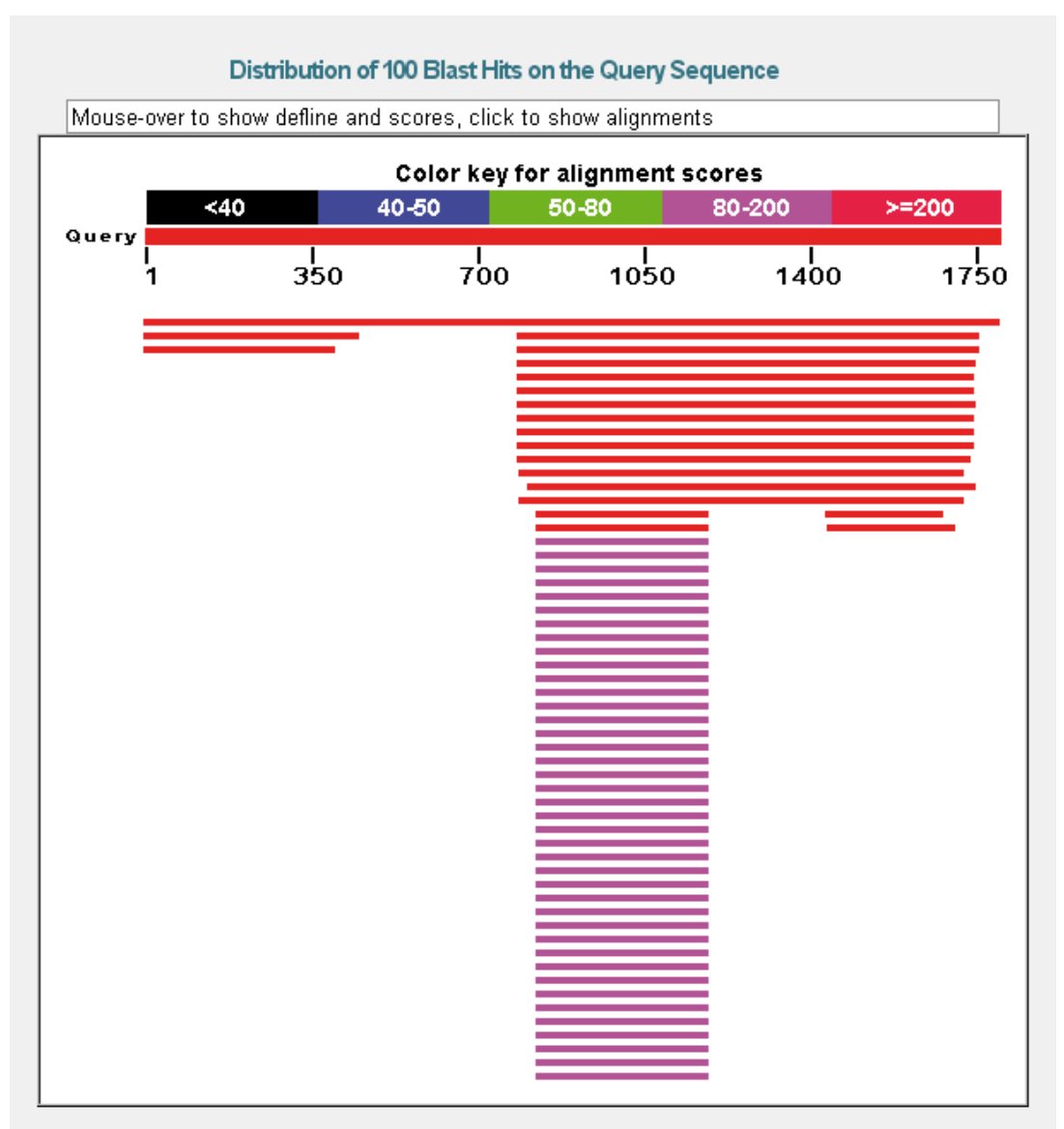

Figure 3. Alignment of Entamoeba histolytica cystein proteinase gene (gi 1246522) with other sequences of cystein proteinase (cds sequences) drawn from the database

On this basis, it can be assumed that there is a non-coding region between 472 and about 700 nt. However, a chosen pair of primers is complement to the fragment of the gene downstream to this site.

GenBank actine gene sequences from Naegleria fowleri (gi|1022820), Entamoeba histolytica (gi|118430606), Acanthamoeba castellanii (gi|5565) and Dientamoeba fragilis (gi|506956256) were aligned. 
nf a

eh_a

ac a

$\mathrm{df}^{-} \mathrm{a}$

$n f \_a$

eh-a

ac_a

df_a

nf_a

eh a

ac a

df $a$

nf_a

eh-a

ac a

df $a$
TTGACTGAAGCTCCATTGAATCCAAAGGCTAACAGAGAAAAGATGACTCAAATCATGTTT TTAACTGAAGCCCCAATGAATCCAAAAGCTAACAGAGAAAAGATGACTCAAATTATGTTT CAGACTGAGGCCCCGCTCAACCCCAAGGCCAACCGCGAGAAGATGACGCAAATTATGTTC CTTACAGAAGCTCCAATGAATCCAAAGGCTAACCGTGAAAAGATGATTCAACTTATGTTC

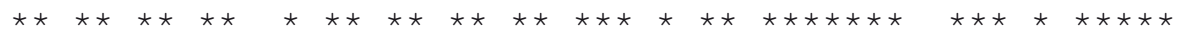

GAAACCTTCTCTGTTCCAGCCATGTATGTTGCCATTCAAGCTGTCTTGTCTTTGTATGCT GAAACATTCAACACCCCAGCTATGTATGTTGGAATTCAAGCTGTTCTTTCATTATATGCC GAGACCTTCAACACCCCCGCCATGTACGTCGCCATCCAGGCCGTGCTCTCGCTCTACGCC GAAACATTCAACACACCAGCYTTYTATGTTGGTATCCAAGCYGTTCTTTCACTTTACGCA

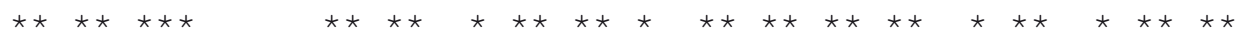
TCTGGTCGTACCACTGGTATTGTTTTGGACTCTGGTGATGGTGTCTCTCACACTGTTCCA TCAGGTAGAACTACTGGTATTGTTATGGATTCAGGTGATGGAGTTTCACACACCGTCCCA TCGGGCCGTACCACTGGCATCGTGCTTGACTCGGGCGACGGCGTCACCCACACCGTGCCC TCAGGTCGTACAACAGGTATTGTTTTCGATGCTGGTGATGGTGTTTCACACACAGTTCCA

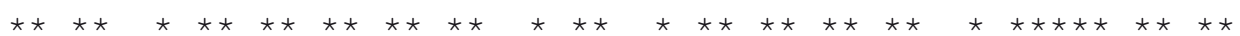
AтtTATGAAGGTTATGCTTTGCCTCATGCTATTTTGAGATTGGATTTGGCTGGTAGAGAT AтTTATGAAGGATTCTCACTTCCACATGCTATTCTTAGACTTGATCTTGCAGGACGTGAT ATCTACGAGGGTTATGCCCTGCCCCACGCCATCCTGCGTCTCGATCTCGCCGGTCGCGAT AтTTATGAAGGTTATTCACTTCCACATGCTATCATGAGACTTAACCTTGCTGGTCGTGAT

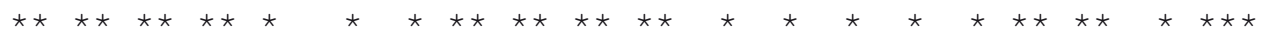

Figure 4. CLUSTAL 2.1 multiple sequence alignment of actine genes

The specificity of primers generated using Primer 3 was authenticated using Primer-BLAST, and the most specific primer pair with the best physical properties was chosen.

\section{¿ Primer-BLAST}

- NCBU//Primer-BLAST : results: Job id=JSID_01_100130_130.14.22.10_9004_primertool more.

Input PCR template AY956428.2 Entamoeba histolytica strain DS6-64 actin gene, partial sequence

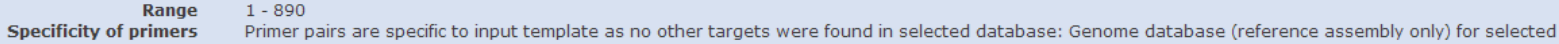
Primer pairs are specific to input template as
species (Organism limited to Homo sapiens)

$>$ Search Summary

$\nabla$ Graphical view of primer pairs

Detailed primer reports

Primer pair 1

Forward primer

Reverse primer

Product length

Sequence $\left(5^{\prime} \rightarrow 3^{\prime}\right) \quad$ Template strand

ACATCAACACCCCAGCTATGTA CATAAATTGGGACGGTGTGTGA

Plus Length Start Stop $\mathrm{Tm}$ GC\% Self complementarity $\begin{array}{llllll}23 & 298 & 320 & 59.73 & 43.48 & 4.00\end{array}$

$421 \quad 400 \quad 58.92 \quad 45.45 \quad 4.00$

Figure 5. Primer-BLAST results for the generated primers

GenBank 5.8S rRNA gene sequences (without the internal transcribed spacer - ITS sequence) from Entamoeba moshkovskii (gi|908849), Entamoeba dispar (gi|1929041), Entamoeba histolytica (gi|1929043) and Entamoeba invadens were aligned.
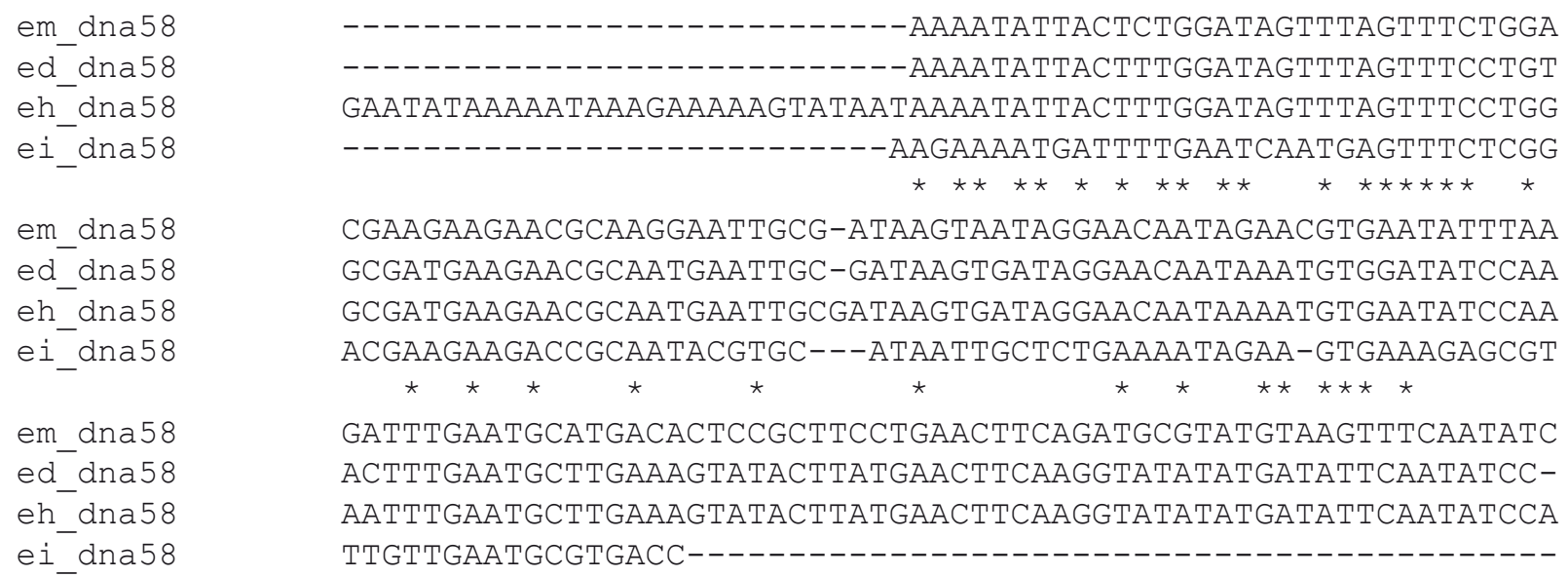

CGAAGAAGAACGCAAGGAATTGCG-ATAAGTAATAGGAACAATAGAACGTGAATATTTAA GCGATGAAGAACGCAATGAATTGC-GATAAGTGATAGGAACAATAAATGTGGATATCCAA GCGATGAAGAACGCAATGAATTGCGATAAGTGATAGGAACAATAAAATGTGAATATCCAA ACGAAGAAGACCGCAATACGTGC---ATAATTGCTCTGAAAATAGAA-GTGAAAGAGCGT

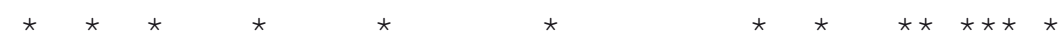

GATTTGAATGCATGACACTCCGCTTCCTGAACTTCAGATGCGTATGTAAGTTTCAATATC

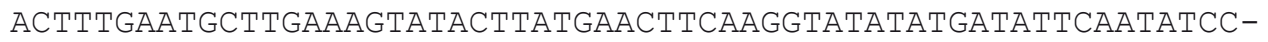
AAтTTGAATGCTTGAAAGTATACTTATGAACTTCAAGGTATATATGATATTCAATATCCA TTGTTGAATGCGTGACC------------------------------------------

Figure 6. CLUSTAL 2.1 multiple sequence alignment without internal transcribed spacers (its) 
WARNING: Left primer is unacceptable: Tm too low/High 3' stability; Right primer is unacceptable: Tm too low/High end self complementarity

OLIGO

LEFT PRIMER

RIGHT PRIMER

53.73

56.80 $\frac{3^{\prime}}{3.00} \frac{\text { seq }}{\mathrm{TTG}}$

$\overline{3.00}$ TTGGATAGTTTAGTTTCCTGG

7.00 TCAAGCATTCAAATTTTGGA

SEQUENCE SIZE: 383

INCLUDED REGION SIZE: 383

PRODUCT SIZE: 96, PAIR ANY COMPL: 4.00, PAIR 3' COMPL: 1.00

1 AgGtgAACCTGCGgAAgGATCATTAAAAGAAAAGAAATAATCTTTTAAAATAAAACGAGA

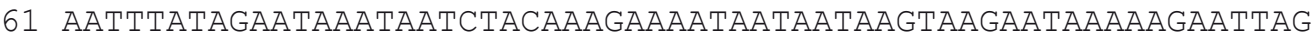

\section{AATATAAAAATAAAGAAAAAGTATAATAAAATATTACTTTGGATAGTTTAGTTTCCTGGG \\ $>>>>>>>>>>>>>>>>>>$ \\ 181 CGATGAAGAACGCAATGAATTGCGATAAGTGATAGGAACAATAAAATGTGAATATCCAAA $<<<<<<$ \\ 241 ATtTGAATGCTTGAAAgTATACTTATGAACTTCAAGgTATATATGATATTCAATATCCAA $<<<<<<<<<<<<<<$

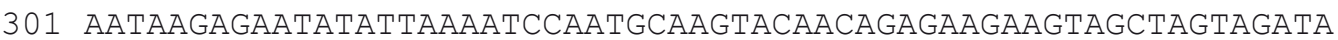

\section{AATGAGAGAAGAAGTAAAGAGCT}

Figure 7. Primer 3 results for primers to 5.8S rRNAThe melting temperature of both primers is considered too low, because of a too low GC content. It is thus, deemed impossible to design primers for the 5.8S rRNA gene with the appropriate parameters

\section{DISCUSSION}

The best pair for cysteine proteinase gene and actine gene identification was chosen after a comparison of sequences and the designing of primers for the most conserved regions. The sequences of primers for cysteine proteinase are as follows: forward - GATTGGAGAGCTGAAGGTAAAGT, reverse - CATGAAGTTCCCCATGAGTTCTTA while that for actin are: forward - ACATTCAACACCCCAGCTATGTA, reverse - CATAAATTGGGACGGTGTGTGA. The primers are specific to the appropriate gene sequence of Entamoeba histolytica. However, they should be examined experimentally in order to be considered suitable for the amplification of relevant Entamoeba gingivalis genes. As the primers may not be completely complementary to $E$. gingivalis genes, a modification of conditions (such as providing annealing temperatures) should be applied.

The primers complement to SSU rDNA and currently used to identify the Entamoeba gingivalis are called EGO-1, EGO-2 $[5,7,10]$. They are characterized by having high selfcomplementarity and 3' end complementarity. This results in a high probability of hairpin and homo and heterodimer formation, as well as difficulties in their usage. Finding primers with good properties would be considered to be important for both epidemiological studies and for diagnostic purposes. Such primers would contribute to greater efficiency of the method.

\section{REFERENCES}

1. Bhaijee F., Bell D.: Entamoeba gingivalis in Acute Osteomyelitis of the Mandible. Case Reports in Medicine, Article ID 357301, 2011.

2. Brown T.A. (2002). Genomes, $2^{\text {nd }}$ edition. Department of Biomolecular Sciences, UMIST, Manchester, UK. Oxford: WileyLiss. Chapter in a book: 3,11 .

3. Derda M. et al.: Incidence of Entamoeba gingivalis in the oral cavity of students. Journal of stomatology, 64, 10: 784-795, 2011.

4. Dominguez R., Holmes K.C.: Actin structure and function. Annual Review of Biophysics, 40:169-86, 2011.

5. Foda A.A., El-Malky M.M.: Prevalence of genital tract infection with Entamoeba gingivalis among copper T 380A intrauterine device users in Egypt. Contraception, 85(1):108-12, 2012.

6. Jian B. et al.: Entamoeba gingivalis pulmonary abscess - diagnosed by fine needle aspiration. Cytojournal., 30 (5):12, 2008.

7. Kikuta N., Yamamoto A., Goto N.: Detection and identification of Entamoeba gingivalis by specific amplification of rRNA gene. Can J Microbiol., 42(12):1248-51,1996.

8. Que X., Reed S. L.: Cysteine Proteinases and the Pathogenesis of Amebiasis. Clin Microbiol Rev., 13(2): 196-206, 2000.

9. Rozen S., Skaletsky H.: Primer3 on the WWW for general users and for biologist programmers. Methods Mol Biol., 132:365-86, 2000.

10. Trim R.D. et al.: Use of PCR to detect Entamoeba gingivalis in diseased gingival pockets and to demonstrate its absence in healthy gingival sites. Parasitol Res., 109:857-864, 2011. 